\title{
Frontières
}

\section{Ils sont feu ces pompiers!}

\section{Nathalie Ferrand}

Volume 12, numéro 2, printemps 2000

Peur bleue...

URI : https://id.erudit.org/iderudit/1074402ar

DOI : https://doi.org/10.7202/1074402ar

Aller au sommaire du numéro

Éditeur(s)

Université du Québec à Montréal

ISSN

1180-3479 (imprimé)

1916-0976 (numérique)

Découvrir la revue

Citer cet article

Ferrand, N. (2000). Ils sont feu ces pompiers! Frontières, 12(2), 64-68.

https://doi.org/10.7202/1074402ar

\section{Résumé de l'article}

Si les pompiers sauvent des vies, c'est souvent au péril de la leur. Ils évoluent en permanence dans des situations à risques de différentes natures. Comment appréhendent-ils ces risques, ainsi que l'angoisse, le stress ou la peur qui en découlent? Nous nous sommes consacrés à un portrait subjectif, saisi du point de vue des principaux acteurs qui nous révèlent leurs manières de parler du feu, de la peur et des situations angoissantes. C'est la structure organisationnelle, unique, du corps des pompiers qui leur permet de contrôler et de partager leurs émotions. L'expérience joue également un rôle important.
Ce document est protégé par la loi sur le droit d'auteur. L'utilisation des services d’Érudit (y compris la reproduction) est assujettie à sa politique d'utilisation que vous pouvez consulter en ligne.

https://apropos.erudit.org/fr/usagers/politique-dutilisation/ 


\section{Résumé}

Si les pompiers sauvent des vies, c'est souvent au péril de la leur. Ils évoluent en permanence dans des situations à risques de différentes natures. Comment appréhendent-ils ces risques, ainsi que l'angoisse, le stress ou la peur qui en découlent? Nous nous sommes consacrés à un portrait subjectif, saisi du point de vue des principaux acteurs qui nous révèlent leurs manières de parler du feu, de la peur et des situations angoissantes. C'est la structure organisationnelle, unique, du corps des pompiers qui leur permet de contrôler et de partager leurs émotions. L'expérience joue également un rôle important.

Mots clés: pompier - contrôle des émotions - feu - angoisse - groupe

\section{Abstract}

If firefighters save lives, it is often at the risk of their own lives. They are forever stepping into situations involving risks of various kinds. How do they interpret these risks as well as the anxiety, stress or fear that stem from these risks? We have devoted ourselves to a subjective portrait, taken from the viewpoint of the main actors, who reveal to us their ways of speaking about fire, fear and distressing situations. It is the unique organizational structure of the fire brigade that enables them to control and share their feelings. Experience also plays an important role.

Key words: firefighter-contro/ over feelings - fire - distress - group

\section{Ils sont feu ces pompiers!}

\author{
Nathalie Ferrand, \\ recherchiste à Télé-Québec.
}

\section{DÉTOUR SANS URGENCE: DE TOUS LES TEMPS, LE FEU, VECTEUR D'HUMANITÉ}

D'aussi loin que l'on observe l'histoire de l'humanité, la lueur d'une flamme rougeoyante éclaire le regard. Car l'histoire de l'humain se confond avec celle du feu, autant en des termes physiques qu'en des termes symboliques. "Selon toute vraisemblance l'utilisation puis la production du feu sont inhérentes à l'apparition du genre Homo [et] elles viennent au premier rang des conditions de sa survie et de son évolution. ${ }^{1 »}$. Et si d'autres animaux et primates sont capables d'utiliser et de produire des outils, la maîtrise du feu est inhérente et spécifique au petit d'homme. Souvenons-nous, un air «jazzé»venu du fin fond de la jungle; c'est Mowgli capturé dans la cité des singes par le roi Louis qui tente de lui soutirer le secret des hommes, le secret du feu. Walt Disney ${ }^{2}$ avait donné la parole aux singes; il ne leur manquait plus que le feu!
Cette forme de captation des forces de la nature par l'homme est la plus ancienne et elle semble suivre la courbe de la naissance et du développement de la conscience. Il fallait d'abord surmonter la peur du feu. L'homme devait alors être conscient. Plus tard, il découvrait le moyen d'entretenir le feu et de l'allumer, devenant de plus en plus conscient. Porteur de chaleur et de lumière, le feu était alors efficace pour la lutte contre les principaux ennemis de nos ancêtres, soient les fauves, le froid et l'obscurité. Ensuite, il fut indispensable pour la cuisson des aliments, pour le travail du bois et de la pierre, et même pour l'art préhistorique qui orne les parois des cavernes. La fabrication des poteries, la fonte et le façonnement des métaux sont autant d'utilisations du feu qui s'inscrivent comme des manifestations de la culture dans l'évolution de l'humain.

Son importance et son mystère donnent rapidement au feu un caractère sacré3. Bénéfique autant que maléfique, le feu se situe au premier rang dans la symbolique universelle: source de vie, de régénérescence, de purification, comme 
emblème des antres infernaux ou arme du dieu justicier. En définitive, les usages du feu dans les différentes civilisations lui ont toujours conféré un statut particulier, parce que ambivalent, parmi les éléments de la nature. Par exemple, ceux qui travaillent avec le feu sont craints autant que respectés pour leur possible relation avec les forces obscures, comme ce fut le cas pour les forgerons du Moyen Âge. En sciences des religions 4 aujourd'hui, le terme "feu» ouvre des perspectives fort riches car

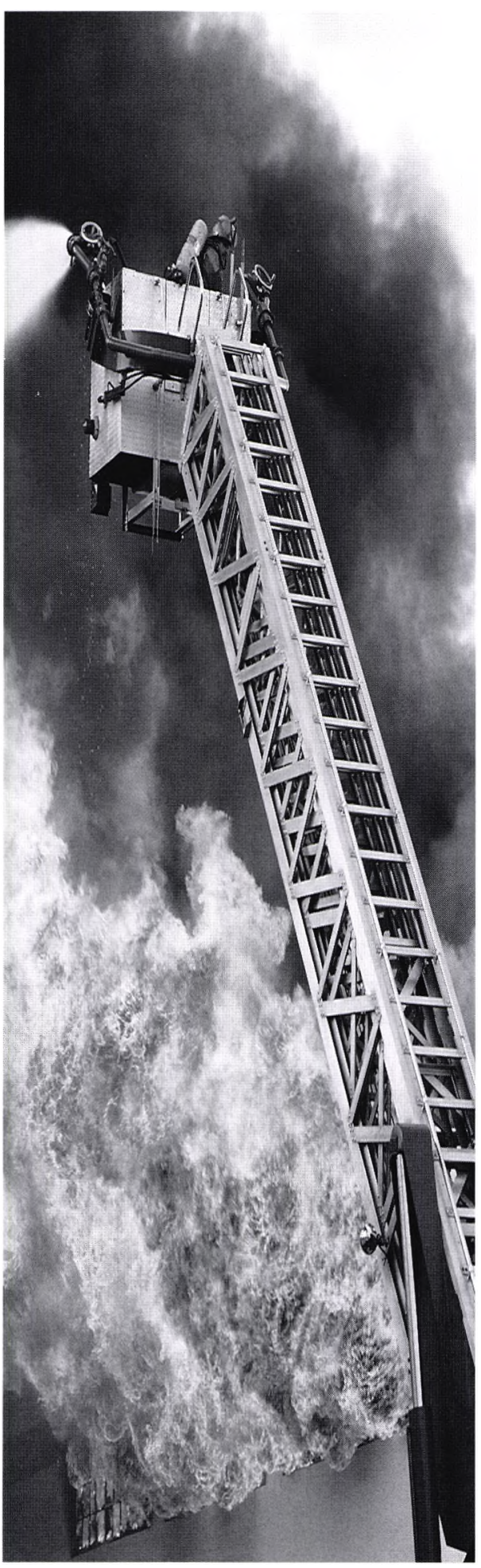

son champ conceptuel et sémantique englobe aussi bien l'utilisation matérielle du feu pour des besoins quotidiens que son emploi liturgique et rituel, sa signification métaphorique, son instrumentalisation magique ou son interprétation ascétique.

Enfin, le feu nous allume jusque dans notre système cognitif lorsque, par exemple, des expressions idiomatiques courantes (la flamme amoureuse, le feu de la colère, etc.), l'assimilent à l'ardeur des sentiments et à l'amour. Outre le rang qu'il tient matériellement et symboliquement dans l'histoire de l'humanité, le feu a aussi une implication sociologique. Ne parle-t-on pas du "foyer» pour parler de la famille? L'emploi des termes "feux" et "foyers" a même servi d'unité démographique lorsqu'on parlait au Moyen Âge d'impôts répartis par "feux», de sociétés de gens vivant "à même feu, pain et sel» ou encore de villages composés de tant de "feux».

Le feu, reconnu dans le soleil, dans la foudre, dans le souffle brûlant des volcans a donc traversé le temps et l'espace pour toujours exercer cette attraction mêlée de crainte dans l'esprit des hommes. Et cela continue.

\section{PLEINS FEUX SUR UNE PRÉSENCE RASSURANTE}

Aujourd'hui, lorsqu'on évoque le spécialiste du feu, on ne pense plus au for geron, à un dieu aztèque ou à une sorcière; c'est la fière allure du pompier qui s'imprime dans notre imaginaire. Le soldat du feu. Et quel soldat! Quel enfant n'a pas un jour rêvé, en se faisant décoiffer par la sirène du camion rouge qui filait à tout rompre sous ses yeux, de combattre les flammes? Quel corps de métier peut se vanter d'avoir une cote de popularité aussi élevée que celle des pompiers? Les pompiers bénéficient en effet d'une image d'autant plus dorée qu'ils sont confrontés au danger et pour une cause on ne peut plus noble: sauver des vies. C'est dans le rapport qu'ils entretiennent avec le feu, les risques, la mort, les émotions des autres et les leurs que le cas des soldats du feu nous a semblé intéressant à plus d'un titre. De ces hommes qui mènent une vie difficile, souvent dangereuse, parfois traumatisante mais ô combien passionnante, excitante, voire envoûtante, voici quelques témoignages.

\section{ENFIN UN INCENDIE!}

25000 appels par année. C'est environ le nombre d'interventions que gèrent le Capitaine Luc Robillard et ses quatorze pompiers à la caserne 16 de la rue Rachel, en plein cœur du plateau Mont-Royal à Montréal. Des appels de détresse, des S.O.S angoissés par la peur des flammes ou par celle d'assister au plongeon de son chaton favori du haut d'une branche; autant de situations mettant la peur, l'angoisse, le stress et la panique au premier rang des émotions vécues. Parmi ces situations, les interventions pour incendie ne représenteraient selon le Service d'incendie de la ville de Montréal que $2 \%$ à $3 \%$ du travail de pompier. "C'est sans compter le temps que l'on passe encore sur les lieux à nettoyer, à chercher la cause de l'incendie ou encore, de retour à la caserne, à nettoyer tout notre équipement», explique le capitaine Robillard qui évalue pour sa part à presque 20\% les interventions «incendie». C'est beaucoup et peu à la fois. Beaucoup en énergie et en émotions mais seulement un cinquième du temps de travail. Cela explique en partie la convoitise du personnel pour ce genre d'opérations. Car le rougeoiement des flammes a toujours exercé un pouvoir de fascination sur l'homme et dans certains cas psychopathologiques limites, des pompiers deviendraient même pyromanes pour le plaisir de combattre l'incendie! Pour messieurs Robillard et Prévost, ce dernier, pompier à la même caserne, les interventions avec du feu sont également leurs favorites. "C'est un challenge plus élevé pour nous de combattre l'incendie que d'aider simplement les gens. Il y a quelque chose d'excitant et d'un petit peu fou aussi », ajoutent-ils. La force de l'émotion grandit alors proportionnellement à l'intensité du risque de la situation. Cardiaques, s'abstenir.

\section{LES VICTIMES VUES PAR LES POMPIERS}

Les victimes, elles, attendent interminablement l'arrivée de leurs sauveurs dans la crainte de perdre tous leurs biens, leur animal domestique et peutêtre leur propre vie. Bref, peu à peu part en fumée sous leurs yeux, tout ce qui donne un cadre à leur quotidien, un lieu à leur identité et à leur mémoire. Les émotions ne sont forcément pas vécues et exprimées de la même manière par les pompiers et par les victimes. Ces dernières ne s'attendaient pas à participer au spectacle flamboyant. L'élément de surprise existe aussi pour les pompiers car chaque situation est nouvelle et chaque incendie a ses propres caractéristiques. Mais quand il est appelé à intervenir sur les lieux d'un incendie, le pompier a derrière lui des années de formation et d'expérience qui lui ont appris à (tout) calculer, à connaître le comportement du feu et à contrôler ses propres 
émotions. Parlant de celles des victimes, le capitaine Robillard modère: "Si la personne a pu nous appeler, c'est qu'elle n'était sûrement pas en danger". Certes! La perception du danger est définitivement différente pour ces combattants.

Ils ont même constaté que le comportement des victimes avait évolué en quelques décennies. Selon le capitaine de la caserne 16, "les comportements de panique se sont accentués; les gens vivent de plus en plus pour eux-mêmes. Chacun a ses biens, chacun a ses choses: "Ce qui se passe pour moi c'est grave, mais pour les autres c'est pas grave." Nous, on ne peut pas être partout à la fois. Si on devait répondre à un feu dans un bâtiment de vingt appartements, il faudrait répondre à un à la fois au lieu que tout le monde se mette ensemble pour s'entraider. Mais on voit de moins en moins ce genre de comportements. Les gens essaient de t'accaparer et tant pis pour les autres. Peut-être qu'avant le monde était moins attaché au matériel... Mais c'est aussi un comportement de ville. En campagne, c'est différent». Quel constat troublant. L'urbattitude..

\section{LA BARRIĖRE DE LA PROCÉDURE}

Et nos pompiers dans tout ça: quel est leur secret pour réussir à composer avec des situations de stress intense? «Quand tu arrives sur les lieux, tu réagis toujours de la même manière. "Où est-ce que l'incendie a été mis, quelle est sa cause, estce qu'il est d'origine naturelle, accidentelle, etc.?" Et si j'arrive chez toi et que, paniquée, tu t'accroches à moi, il ne faut pas que je m'arrête à ça; je vais te confier à quelqu'un d'autre. Il faut se faire une barrière parce qu'on a beaucoup de choses à faire quand on arrive sur les lieux d'intervention". La réponse du sauveteur face à l'urgence et à l'intensité émotionnelle de la situation, c'est «la barrière" qui équivaut à la procédure. "C'est tellement automatique; il y a tellement de choses à vérifier, tellement de choses à s'assurer, que c'est rendu comme un boulot sur une ligne de montage». Procédure: c'est le mot magique qui offre le recul nécessaire pour être efficace afin d'agir envers les victimes, en fonction de l'équipe et pour sa propre sécurité, physique et mentale. La procédure étouffe la peur autant qu'elle le peut, et de mieux en mieux avec le temps.

\section{DU STRESS FLAMBANT NEUF À L'EXPÉRIENCE}

Mais la première fois, c'est toujours terrible. Souvenez-vous de votre première intervention Monsieur le pompier: vos genoux tremblaient comme des feuilles quand vous étiez debout à l'arrière du camion et arrivé sur place, à vouloir tout faire vous n'avez servi à rien, complétant le tableau en trébuchant par trois fois sur les boyaux au sol!

Pour tout pompier, le "baptême du feu " est en effet un événement important, impressionnant, à la fois désiré et redouté. Mais le "mal aux tripes", les genoux qui sautent, le manque de coordination et tous les autres symptômes s'amenuisent avec le temps sans pour autant disparaître complètement. "Aujourd'hui, j'en vis tout de même, des stress; c'est peut-être plus interne qu'externe. Mais à toutes les fois, j'ai un stress". Le retour des émotions vers leur origine, un lent cheminement de l'extérieur vers l'intérieur, ne laissant transparaître que de faibles manifestations de l'adrénaline: c'est le résultat de l'apprentissage du contrôle de soi par l'expérience.

"Mais si on n'est pas nerveux, c'est qu'on ne veut pas performer» de rajouter le capitaine. "C'est un bon stress si t'es capable de le gérer. Parce que tu feras davantage en état de stress que ce que tu ferais normalement. Et la journée où je ne l'ai pas, c'est là que je serais dangereux pour quelqu'un». Lorsqu'elle ne provoque pas l'absence totale de réaction, la peur comporte des aspects constructifs et créatifs pour ceux qui la vivent autant en termes individuels qu'organisationnels. La situation d'urgence que représente un incendie est par exemple un facteur de division du travail. Avant on faisait la chaîne pour transporter davantage qu'un seul seau d'eau; aujourd'hui on divise toujours le travail et la prise en charge des victimes fait partie de l'une des nombreuses tâches que l'équipe doit organiser et exécuter.

\section{SOLIDARITÉ ET FAMILIARITÉ DES ÉMOTIONS: SA PEUR POMPIER}

L'équipe; c'est aussi cet autre élément très important qui rend cette "barrière" possible et souhaitable devant le déferlement d'émotions que provoque la situation d'urgence. Les mots "pompier» et «solitude» sont antinomiques. Un pompier combat les flammes en équipe, un pompier mange en équipe, dort en équipe, vit son quotidien en équipe. Les liens qui unissent les hommes d'une caserne sont de nature autant professionnelle, sociale, qu'affective; ils les qualifient eux-mêmes de "familiaux». "On passe plus de temps avec le personnel ici qu'avec notre propre famille; il y a des choses qu'eux savent sur ma vie privée que ma femme ne sait pas". Cette relation privilégiée permet davantage de contrôle lors des interventions car il devient (presque) possible de prévoir le comportement des hommes en toutes situations. La grande connaissance de soi et des autres est en effet indispensable pour créer les conditions de la confiance nécessaire à l'action adéquate et mesurée. "On dépend tous les uns des autres avec des tâches très précises à effectuer».

De cette codépendance naît une grande transparence de l'autre et de ses états émotionnels. "On sait si une personne vit des émotions; on va le ressentir tout de suite dans les situations de stress. Tu t'attends à une réaction $\mathrm{x}$ d'une personne et celle-ci ne réagit pas comme tu t'y attendais. Là tu te dis, «il y a quelque chose qui ne fonctionne pas; il a peut-être vécu quelque chose ou vu quelque chose que je n'ai pas vu.» Mais il faut que je sache ce qui se passe. S'il n'a pas réagi, il faut qu'il m'explique pourquoi afin que tout le monde puisse travailler ensemble" renchérit le capitaine. D'individuelle, l'émotion devient alors rapidement collective. Qu'elle soit due à une situation d'intervention dans laquelle un citoyen ou un collègue fut très affecté voire mortellement atteint ou tout simplement à un départ à la retraite d'un ancien, elle se vit en groupe.

\section{POST MORTEM ET PARTAGE SOCIAL DES ÉMOTIONS}

Ce vécu de groupe nécessite aussi de prendre en compte les conséquences des émotions vécues à l'intérieur du groupe. Jean-Pierre Bergeron, instructeur-pompier depuis 10 ans, enseigne parmi d'autres modules, celui intitulé "Psychologie en situation" à l'Institut de protection contre les incendies du Québec. Il est formel: "Les notions enseignées dans ce cours sont très importantes. On apprend aux pompiers à rester vigilants face aux demandes parfois déraisonnées des victimes et on leur parle surtout des symptômes post-traumatiques et des règles et effets du debriefing». Car même si on prend en compte les aptitudes de chacun, il n'y a pas de pompier spécialement mandaté pour traiter les réactions des victimes. Chacun doit en théorie pouvoir tout faire et remplacer l'autre. En ce qui concerne le debriefing ou post mortem, c'est une procédure courante qui vise à diminuer les séquelles psychologiques du traumatisme. On encourage alors ceux qui sont intervenus à s'exprimer, en huis clos, à propos de leurs perceptions de l'intervention et de ce qu'ils ont ressenti. Curieusement ce n'est pas le service permanent d'un psychologue affecté à 
cette tâche qui est offert aux pompiers. Lorsqu'une situation de crise est jugée suffisamment grave pour nécessiter un debriefing, le service d'incendie appelle un psychologue, préférablement toujours le même, qui se rend aussi vite que possible sur les lieux pour intervenir directement auprès des pompiers. Car les séquelles d'un vécu douloureux peuvent parfois prendre des décennies avant de se révéler comme telles. "J'ai vu un collègue se couper la moustache car une odeur infâme qui l'avait affecté semblait s'accrocher depuis longtemps à son nez; un autre voyait le corps d'un enfant qu'il avait secouru dans sa baignoire à chaque fois qu'il prenait son bain". C'est pour cette raison que $\mathrm{M}$. Bergeron rappelle en tout temps à ses collègues que ses oreilles ont le dos large.

$\mathrm{Si}$ à un moment $\mathrm{x}$ un pompier demande à consulter un psychologue, cette requête suit le long processus administratif des supérieurs avant d'aboutir. Les post mortem ne sont donc pas systématiques, même s'ils sont de plus en plus fréquents depuis une dizaine d'années. Les pompiers assistent aussi de plus en plus souvent à des séminaires où des personnes ressources viennent les entretenir de la nécessité de partager, de verbaliser leurs émotions le plus rapidement possible. À Québec, le premier pompier arrivé sur les lieux de l'écrasement de l'avion F275 de la compagnie Québecair le 29 mars 1979, continue de parler aux plus jeunes de cette expérience traumatisante et de ses séquelles... Et dans la salle, l'émotion se transmet; le récit ${ }^{6}$ de telles expériences a un impact affectif énorme. Il suscite l'intérêt, l'empathie et l'attirance dans le silence le plus envoûtant. Selon Bernard Rimé, professeur de psychologie à l'Université de Louvain en Belgique, "l'apport du partage social de l'émotion à la mémoire est loin de se limiter au niveau individuel7 ${ }^{7}$.". Par le processus du partage social secondaire et tertiaire, c'est la communauté qui entoure l'individu qui apprend ce qui lui est arrivé et donc, ce qui pourrait arriver à l'un de ses membres. Ce partage enrichit alors le savoir adaptatif de la communauté... des pompiers en l'occurrence.

\section{"ÉCOUTEZ, LES GARS..."}

En parlant de debriefing, l'instructeur-pompier rappelle aussi très souvent ses règles élémentaires comme celle qui consiste à «ne jamais chercher le coupable», tout en essayant de décortiquer la situation dont il est question. La chose à ne pas faire, confirme le capitaine Robillard, "c'est de se mettre le tort sur soi. Si l'un de mes gars décède, tu remets tout en question de ce que tu as fait sur cette intervention. Et même si ça peut être utile de diminuer la pression en la donnant à quelqu'un d'autre (en consultation), on la garde toujours. On se dit sans cesse qu'on aurait dû faire ça ou ça pour le sauver». Tout remettre en question et vouloir donner sa démission est une réaction qui arrive fréquemment après un traumatisme. Dans ce cas, $\mathbf{M}$. Bergeron écoute ses collègues se vider le cœur et leur demande de prendre le temps de réfléchir. Peu d'entre eux passent finalement à l'acte.

Lorsqu'il vit une intervention où un collègue est victime d'un accident, la première question que le pompier Prévost se pose est: «est-ce que j'ai fait mon possible pour ne pas que ça arrive? Si on a fait ce qu'on a pu, alors quand c'est fait, c'est fait». "Moi je deale bien avec ça» conclut-il avant de rajouter qu' "un service d'aide psychologique est offert si tu veux». Il poursuit: "ça aurait été il y a 20 ans, ça aurait été la risée; on aurait pas été des hommes d'avoir à en parler. Maintenant on se fait debriefer, quand on est dans le besoin. Ça ne fait pas longtemps, à peine dix ans". Tout en reconnaissant la nécessité d'un tel service ou d'une formation comme celle dispensée dans le module de "psychologie en intervention", les pompiers ne sont pas tous nécessairement à l'aise à l'idée d'étaler leurs états d'âme. "C'est le problème d'un milieu d'hommes" explique M. Bergeron, "les gars ne vont pas tout dire, ils peuvent prendre ça à la légère ou être gênés d'en parler».

Un milieu d'hommes, de soldats qui mènent un combat contre l'incendie (comme le nom du cours "combat incendie» que les pompiers suivent). Avec un vocabulaire guerrier, comment dit-on le mot peur? On ne le dit pas ou peu. On se contente de l'observer rapidement au passage, de la refouler, pour passer à l'action. Pour François Prévost, la distinction est importante: "La peur c'est quelque chose, être effrayé c'est autre chose, contrôler ses émotions c'est encore autre chose. Pour moi la peur, c'est être saisi, c'est se figer». Et ça, les pompiers n'y ont pas droit. C'est le privilège des victimes. Qui aurait envie d'être sauvé par un pompier peureux, figé sous son casque, fondant comme un popcicle sous un soleil brûlant? Personne, et de toutes façons, il n'existe pas. Un pompier parle de stress, d'angoisse, mais pas de peur. C'est un mot qu'il ne prononcera pas. Peut-être parce que comme disait Jean Giono ${ }^{8}$, «il ne faut pas avoir peur; c'est la peur qui tue»... Et même chez les pompiers la mort reste un tabou ${ }^{9}$.

\section{UN FEU TERRIBLE, À DÉCODER}

Mais quittons le domaine intérieur pour parler de ce qui flambe à l'extérieur. Comment décrit-on un feu terrible en parler-pompier? C'est l'enfer, le four, ou la bête... Et la bête, ils vont lui faire mal, la mater, la noircir, la taper. Parce que le feu, c'est un livre pour les pompiers. "Quand on entre dans une pièce en feu, le feu nous parle en même temps qu'on réagit. Quand on fait des mouvements dans la pièce, il nous le renvoie; si on fait des déplacements d'air, il va se propager ailleurs, on le voit, on le lit le feu. C'est comme une histoire pour nous, tu sais comment il réagit, où est-ce qu'il est dans la pièce, tu sais si il y a vraiment beaucoup de gaz chaud dans la pièce. Si j'ouvre une lance dans une pièce et que la chaleur se dissipe, c'est ouvert ailleurs, donc le feu va se propager ailleurs". Alors pour le combattre, le défi consiste à apprendre à le lire. "Quand tu entres dans une pièce tu penses que tu en es le maître; mais tu vois que ce n'est pas toi qui mène, c'est le feu. Si toi tu ne joues pas avec lui dans le sens où il te mène, il n'y a rien à faire tu vas te faire mal». On ne peut pas aller contre la nature, juste l'accompagner, et pour ça, "l'expérience est indispensable. Les réactions du feu ne s'apprennent pas en lisant un document".

Le pire, lorsque le feu prend le dessus, c'est pour le capitaine "lorsqu'il y a des vies en danger. Quand tu vois des gens accrochés par le bout des doigts sur les joints de briques à l'extérieur, la flamme au derrière... Là, ton temps de réaction qui est d'habitude de deux secondes vient de passer à moins quatre secondes. $\mathrm{Tu}$ n'as plus de temps de réaction: tu voudrais en sauver quatre et tu as deux mains, ça c'est le pire». Pour M. Prévost la situation la plus pénible, "c'est quand il $\mathrm{y}$ a des collègues qui sont pris dans l'incendie; là, ça change les données, ça vient d'atteindre un autre degré de stress. C'est vraiment de la folie. C'est le paroxysme». Dans ces deux exemples de situations terrifiantes, on constate que l'émotion ne vient pas directement du danger que le pompier court en tant que tel. Indirectement, bien sûr le pompier est dans une situation à risque, mais ce qui le fait bouillir c'est le danger que vivent les autres; l'émotion grandissant proportionnellement au degré d'attachement qu'ils éprouvent envers les victimes. Vivre de telles situations d'angoisse a-t-il une influence sur le fait d'apprécier la vie ou au contraire de s'en écœurer et de vouloir la quitter? 


\section{LE RISQUE OU LA VIE}

Lors des 450 incendies mortels que le Québec a connus entre 1992 et 1998, vingt pompiers sont morts dans les flammes. En 1998, quatre-vingt-six personnes ont perdu la vie ${ }^{10}$. Le fait d'être perpétuellement confronté au danger, à la peur indicible et à la mort ne contribuerait-il pas à donner des idées suicidaires? Aucune étude ne semble démontrer une telle hypothèse et il n'y a pas de statistiques disponibles étant donné que la profession des victimes de suicide reste une donnée confidentielle. De plus, il n'existe pas de perspectives théoriques bien établies qui peuvent rendre compte des rapports complexes que les comportements à risque entretiennent avec la mort, les conduites suicidaires et le suicide $^{11}$. Cependant, peut-on réellement parler de comportement à risque dans un métier où le moindre geste est méticuleusement calculé, étudié, analysé, vérifié et où rien n'est laissé au hasard? Le métier de pompier est davantage un "métier à risque» qu'un "métier à comportements à risque». Et le rapport existant entre le suicide et le métier spécifique de pompier semble assez faible, selon Jean-Pierre Bergeron: "tout simplement parce que nous sommes très liés». Une fois de plus, la force des liens du groupe joue donc un rôle prépondérant dans le comportement des pompiers par rapport à leur gestion du stress, des symptômes post-traumatiques et de l'idée du suicide. Le modèle organisationnel du corps des pompiers fondé sur une hiérarchie de type autoritaire et un partage d'un large éventail de situations crée donc des liens suffisamment solides au sein des casernes et de la profession pour soutenir le moral des troupes.

En plus du goût de vivre qu'ils cultivent, les pompiers ont une seconde nature: l'instinct pompier. Le capitaine Robillard explique qu'il se surprend luimême dans sa vie de tous les jours. "Si quelqu'un s'étouffe ou si un accident se passe devant moi, je vais réagir de suite. Ça devient naturel; on identifie les mouvements à risque et on les prévient, par exemple. On pense à rien et on va faire les gestes qui sauvent. C'est pas qu'on aime ça se mettre dans la $\mathrm{m}$.... mais on aime ça gérer, contrôler». L'instinct pompier ne les immunise pourtant pas contre le feu et c'est avec une certaine ironie que le capitaine se souvient de la caserne 26 sur la rue Mont Royal qui est passée au feu. "Ils étaient sortis sur une intervention et le feu a pris quand ils n'étaient plus là. On est allé l'éteindre... Ça montre qu'on est comme tout le monde!» Et il rajoute, "on est peut-être les pires aussi. Les cordonniers sont les plus mal chaussés; nous on gère le feu à la légère. Moi je suis correct, mais toi, si tu allumes un feu de camp tu vas faire très attention; nous on sait comment ça allume, alors..." (Il prend moins de précautions) Qui a dit que le pompier n'est pas un flambeur? Ou à tout le moins, qu'il ne déteste pas jouer de sa toutepuissance (fantasmée...)?

En résumé, rappelons qu'un pompier n'agit jamais seul, ni en situation d'urgence, ni dans son quotidien à la caserne. La structure organisationnelle du corps des pompiers offre en effet à ses hommes un partage des émotions de la vie quotidienne, créant ainsi des liens privilégiés unissant le groupe et la communauté. Ce cadre leur permet alors d'endurer les situations stressantes et angoissantes auxquelles ils sont soumis, pendant qu'ils sont aussi protégés par l'aspect très procédurier de leur travail. Pour maîtriser l'appréhension des situations dangereuses, l'expérience et le partage des émotions au sein du groupe sont alors indispensables pour assurer la santé physique et mentale de nos sauveteurs. Car un épisode émotionnel a un impact affectif, mais il a également un impact sur nos systèmes cognitifs. Il devient comme une brèche dans notre représentation rassurante et cohérente du monde. C'est donc pour colmater cette brèche au plus vite afin de ne pas risquer de faire s'effondrer le reste de l'édifice symbolique que le partage social de l'émotion intervient. Il a pour but de "chercher le réconfort et le consensus social ${ }^{12}$ ».

Pourtant, nombre de pompiers se contentent encore de la seule gratitude des citoyens. "C'est pas tant de se sentir aimés, mais appréciés. Tu fais quelque chose dans la société qui rend service aux gens». Un grand merci messieurs!' ${ }^{13}$.

\section{Notes}

1 Pierre GRAPIN, Les incendies, Que saisje, no 1783, 1979. Paris, PUF, p. 10.

2 Dans l'adaptation du Livre de la jungle en dessin animé

3 "Si grandc est la disproportion existant entre la puissance du feu et celle de l'Homme que l'utilisation en apparut vite comme une sorte de vol commis par celui-ci au préjudice des dieux; ainsi l'exprime le mythe de Prométhée, enchaîné, lc foie éternellement dévoré par un aigle, et puni sans doute par où il avait péché puisqu'on peut voir dans ce mode singulier de châtiment la traduction mythiques des effets digestifs résultant du passage de la nourriture crue aux aliments cuits...", Pierre GRAPIN, ibid.. note 1, p. 11.

4 Une présentation d'ensemble des différentes perspectives du "feu» en histoire et en phénoménologie des religions se trou- ve dans: Jean-Pierre BAYARD, La symbolique du feu, Paris, G. Trédaniel, 1992 (1re édition 1986)

5 L'avion, en direction de Montréal, est tombé quelques minutes seulement après son départ de l'aéroport de Québec, causant la mort de 17 personnes en plus d'en blesser sept autres

6 Bernard RIMÉ. "Faut-il parler de ses émotions?", Sciences Humaines, no 104. avril 2000, p. 16-20.

7 Ibid., p. 20.

8 Tiré du dialogue du film Le Hussard sur le toit, réalisé par Jean-Paul RAPPENEAU. France, 1995, 124 minutes, adapté de l'ouvrage du même titre de Jean GIONO. Gallimard. Dans un contexte de panique collective devant le choléra. l'histoire met en scène Pauline et Angelo, deux êtres échappés de Manosque, une ville atteinte: "(Angelo): Moi aussi, je viens de Manosque... N'ayez pas peur, c'est la peur qui tue. [...]. (Pauline): J'ai attendu autant que j'ai pu... J'aurais dû... C'est ma faute s'il lui est arrivé un malheur... (Angelo): Il reviendra. Les hommes comme lui ne meurent pas du choléra, vous savez. Au moins, lui, il sait pour qui il vit. On meurt quand on veut bien.»

9 N.D.L.R.: on observe ici la relative banalisation du terme "angoisse", alors qu'elle est tout de même cliniquement plus grave Inversement. la notion de peur est évacuée d'emblée, méconnaissant sa réalité dans la formation du courage. Mais la peur resterait quand même euphémisée sous le terme "stress", socialement plus valorisé. N'y aurait-il pas dans cette résistance à dire la pcur un stéréotype de virilité et d'hérö̈sme? Quant au tabou de la mort, il est de ces situations, comme ici, où il reste nécessaire pour assurer la cohésion du groupe et la vitalité psychique de ses individus

10 Statistiques de 1997 et de 1998, documents réalisés par la Direction de la sécu rité incendie, Direction générale de la sécurité civile et de la sécurité incendie, Ministère de la Sécurité publique du Québec.

11 Joseph LÉVY, "Les conduites à risque sont-elles des conduites suicidaires?", Frontières, vol. 12, no 1, automne 1999, p. 40 .

12 Bernard RIMÉ, Faut-il parler de ses émotions?, Sciences Humaines, no 104, avril 2000, p. 20.

13 N.D.L.R.: dans l'ambivalence destruction/regénération par le feu, l'expression artistique offre un magnifique exemple contemporain dans "Flamma, Flamma, the Fire Requiem", de Nicholas LENS Pour ce qui concerne la peur d'un feu total, voir: Louis-Vincent THOMAS, «Le sentiment de la mort nucléaire", Communications, no 57, "Peurs», Paris, Seuil, 1993, p. 101-120. 